South African Journal of Geomatics, Vol. 5. No. 3, November 2016

\title{
A Comparative Analysis of Extracted Heights from Topographic Maps and measured Reduced Levels in Kumasi, Ghana.
}

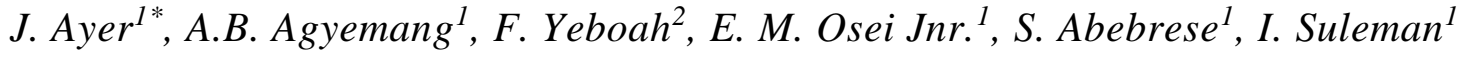 \\ ${ }^{1}$ Department of Geomatic Engineering, KNUST, Ghana. \\ ${ }^{2}$ Building and Road Research Institute, CSIR, Ghana. \\ *Corresponding authors email: jayer.soe@ knust.edu.gh \\ http://dx.doi.org/10.4314/sajg.v5i3.4
}

\begin{abstract}
No other mapping product has had widespread applications in developmental planning than the topographic map. Topographic maps represent the three-dimensional landscape by providing relief information in the form of contours in addition to plan information on which natural and man-made landmarks are quite accurately represented. Height information, extractible from topographic maps, comes in handy for most land use planning. However, generalizations during contouring and the need to interpolate between successive contours for specific grid nodes introduce errors in extracted heights. There is therefore, the necessity to use some mathematical modeling to remove discrepancies in the interpolation process to improve elevation data extracted from topographic maps. In this study, the accuracy of spot heights derived from interpolated and extracted heights from topographical maps is assessed. Two different mathematical models - a third degree polynomial regression model and the Thompson's Multiple Variable Polynomial regression models, were respectively used to model the relationship between extracted heights and ground reduced levels. Results from the two models indicate that, the latter presents better refinements to converting extracted heights into reduced levels with a coefficient of determination value of $95.9 \%$, although further research is recommended to investigate numerical techniques that could improve the solution to the Thompson's polynomial. The Thompson's model was implemented as a crude height refiner program that receives extracted heights to return corrected heights. The implication of these results for the mapping community is that, it is possible to model a correction function that can help obtain reasonably
\end{abstract}


accurate heights from topographic maps. This will reduce the necessity of always going back to the field for field surveys in spite of the fact that topographic maps covering an area already exists.

\section{Keywords}

Topographic maps, Interpolation, Thompson's polynomial, Levelling, Terrain models

\section{Introduction}

Topographic maps represent the three-dimensional landscape with relief information in the form of contours, useful for Digital Elevation Modeling (DEM) and are quite indispensable in a National Geospatial data infrastructure for most development planning. They further quite accurately, represent natural and man-made landmarks and allow for modeling and visualizing of areas at risk from natural hazards (McChesney and McSweeney, 2005). Height information extractible from topographic maps, if accurate enough, could be used for planning the design of roads and railways, ports and dams, calculating quantities of excavation and backfills, studying soil erosion and surface drainage, for irrigation projects, and for mineral exploration.

Traditionally height data is obtained from leveling campaigns which results in reduced levels of points. However, the process of leveling is quite laborious, time consuming and expensive usually requiring field observations followed by rigorous post field computations. A minimal cost solution for generating DEMs consists in interpolating elevation values between contours from digitized topographic maps. The process of extracting heights from contours on existing topographical maps and integrating same with leveled spot heights can be problematic due to generalizations during contouring, and the need to interpolate between contours for specific grid nodes. There is therefore, the necessity of some mathematical modeling to remove discrepancies in the interpolation results.

Though there has been extensive work on the application of different spatial interpolation methodologies to represent landforms (Erdogan, 2009), little if any, research has been done on the reverse process of extracting and comparing heights from contours or surfaces such as TINS to reduced levels of points. A method of heightening from stereo pairs of photographs have been 
developed using a parallax bar in which corrections of a parabolic and hyperbolic combination are applied to any crude heights to obtain near true reduced levels using an equation of the form $a_{0}+a_{1} x+a_{2} y+a_{3} x y+a_{4} x^{2}$

where $\mathrm{x}$ and $\mathrm{y}$ are the planimetric coordinates and $a_{0}, a_{1}$ etc. are constants (Thompson, 1968; Methey, 1970).

Our approach in this study is to investigate a possible model for obtaining real heights from such extracted crude heights with the ultimate goal of using the contours to obtain near true heights by adopting the photographic height extraction model. This research hence, presents a framework for correcting interpolated spot heights from contours into reduced levels.

\section{Concepts in terrain modeling and interpolations.}

Extraction of spot heights from contours

Contour lines are drawn on maps to connect points of equal elevation. To enhance legibility, topographic maps show lines for evenly spaced elevations at a constant vertical distance known as the contour interval. Consequently, whereas heights of points on a contour line can be easily extracted, elevations of points in between two consecutive contours have to be obtained through interpolation, which may not coincide with their exact heights.

Determining ground reduced levels from contour drawings involve the recreation of a grid to be superimposed on the contours to identify grid intersections that contour lines would pass through and assigning the height values of these contours to the respective grid intersection points. However, spot heights may also be required for points through which contours would not be passing. Therefore, surfaces must be created first with interpolated height values for every point across the extent of the surface derived from the limited set of sample values measured. After a suitable surface has been created with interpolated values for heights, the imposed grid can have interpolated height values for each intersection as required. Different interpolating tools used to create a continuous surface from contours would influence the resulting surface differently (Childs, 2004; Tomlison, 2007). 
Each of the many available interpolation methods could work best for a particular data set so there may be no particular interpolation method which would guarantee the best results for all data sets (Erdogan, 2009). Further, different methods of interpolation would produce different estimates for height values at the same point (Fisher and Tate, 2006). Therefore, the choice of spatial interpolation must depend on the planimetric (x,y) and the topographic (z) accuracy of the data; the spatial arrangement and density of the data; prior knowledge of the surface to be interpolated; and the accuracy requirements of the surface being interpolated (Collins, 1995).

The Inverse distance weighting (IDW) deterministic interpolation method calculates values at un-sampled points from known point values using a weight function in a search neighborhood so that points closer to the predicted area have more influence than points of further distance ( Johnston et al., 2003). Its main disadvantage is that the choice of weighting function could introduce ambiguity resulting in smoothing uneven surfaces.

Splines or radial basis functions (RBFs), are inappropriate for producing surfaces from datasets that are prone to error and also datasets with large or rapid changes in the surface values (Godone and Garnero, 2013).

The Kriging geostatistical interpolation method based on statistical models including autocorrelation is a weighted moving averaging method of interpolation derived from regionalized variable theory which assumes that the spatial variation of any property is statistically homogenous throughout the surface and this spatial variation being expressed in terms of semi-variograms from which the weight function is derived (Englund and Sparks, 1988). Though the more the sample points, the more accurate the empirically derived variogram function, and thus rendering more accurate estimates, the original data points are seldom honored (Englund and Sparks, 1988).

The Natural Neighbour (NN) interpolation method uses only a subset of samples that surround the point of interest to generate local surfaces based on Voronoi tessellations (Bater and Coops , 2009). Natural neighbour performs well for irregularly distributed data (Gold , 1989; Sambridge et al., 1995; Watson and Philip , 1987). The neighbours used in the estimation are selected using the adjacency relationships of the Voronoi Diagrams and would always result in the selection of neighbours that both surround and are close to $\mathrm{x}$ with an interpolation function 
$\mathrm{f}(\mathrm{x})$ at the location $\mathrm{x}$ given by $f(x)=\sum_{i=1}^{k} w_{i}(x) a_{\tilde{i}, \ldots \ldots \ldots \ldots \ldots \ldots \ldots \ldots \ldots \ldots \ldots \ldots \ldots \ldots \ldots} \ldots \ldots \ldots$ (Sibson, 1981).

where $w_{i}$ are the weights and $a_{i}$ are the known data at position $\mathrm{x}$.

In contrast to other methods, the NN interpolation is not affected by the regularity or nonregularity of data. This therefore, limits anisotropic issues since the selection of the neighbours is based on the configuration of the data itself and hence, the most appropriate when sample data points are distributed with uneven density as in the present study.

\section{Materials and methods}

Topographic digital data at source scale of 1: 2500 covering major towns in Ghana have been compiled from photographic mapping by the Survey and Mapping Division (SMD) in 2014. Twelve of such map sheets cover the Kwame Nkrumah University of Science and Technology (KNUST) campus which is the study area for the current investigation. Reduced levels of some four thousand (4000) points spread over the campus through spirit leveling process are also available for comparison with those extracted from digital topographic town sheets.

The KNUST Campus is located in Kumasi, the Ashanti Regional capital of Ghana and is Ghana's foremost Institution for Science and Technology education. The Campus covers a land area of approximately $16 \mathrm{~km}^{2}$ undulating land, and is located between latitudes $6^{\circ} 39^{\prime} 42^{\prime} \mathrm{N}$ and $6^{\circ} 41^{\prime} 44^{\prime \prime} \mathrm{N}$ and longitudes $1^{\circ} 31^{\prime} 56^{\prime \prime} \mathrm{W}$ and $1^{\circ} 35^{\prime} 22^{\prime}$ W.

The methodological approach used in this study is summarized in Figure1 below. 


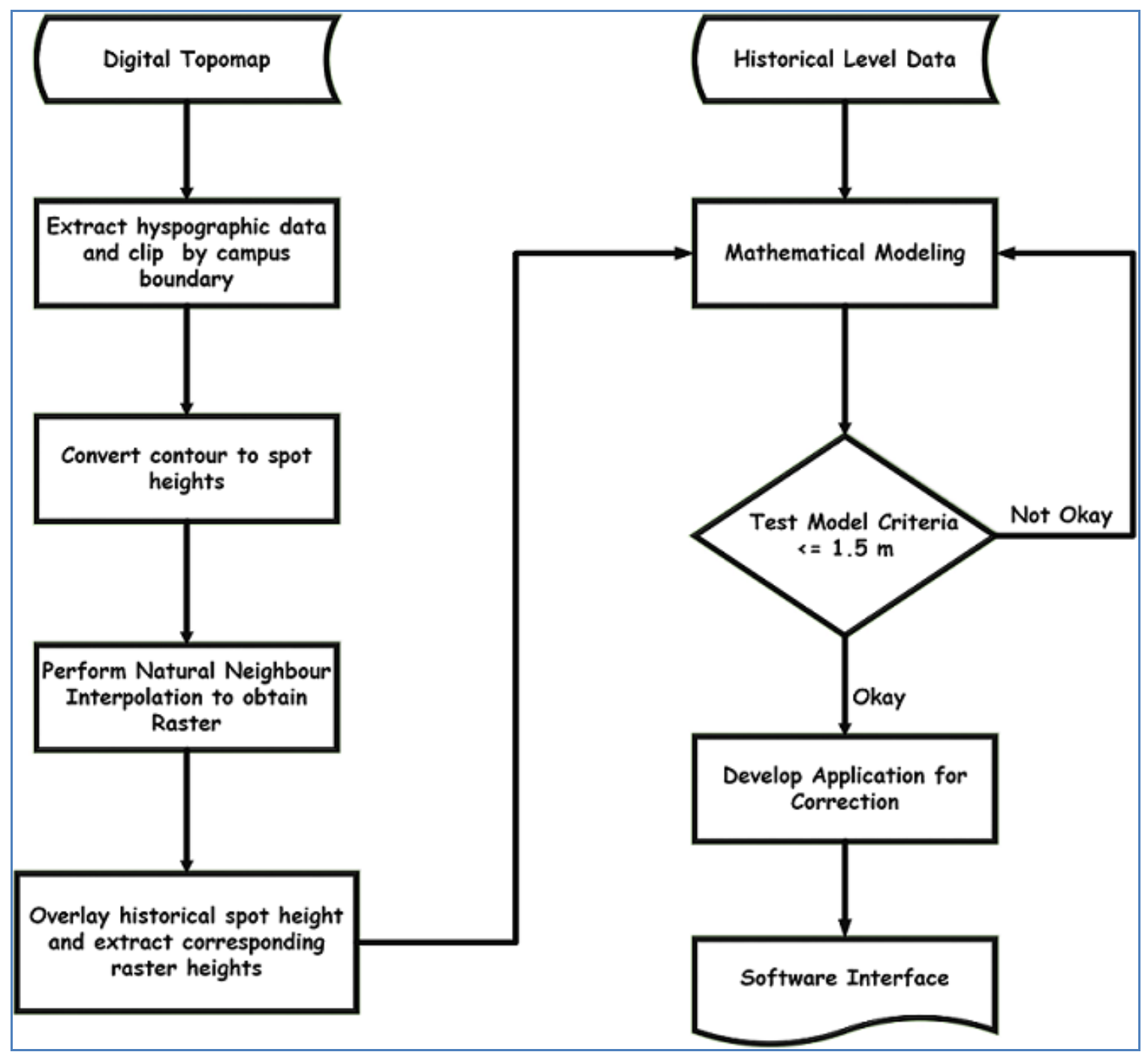

Figure 1: Methodology flowchart

The datasets of the topographic town sheets were exported to shape files from the original Arc Info interchange file format (E00). They were then clipped out with the campus boundary coverage to obtain a contour map for the study area. The contour map was now converted to point data. ArcGIS has tools that can generate a specified number of randomly-placed points on a selected feature layer. This tool was used to create hundred (100) randomly placed points on each contour (Figure 2.), which were then serially numbered for later identification using the ArcMap Field Calculator. The plannimetric coordinates of each point was also added to the attribute table within the ArcMap environment. 


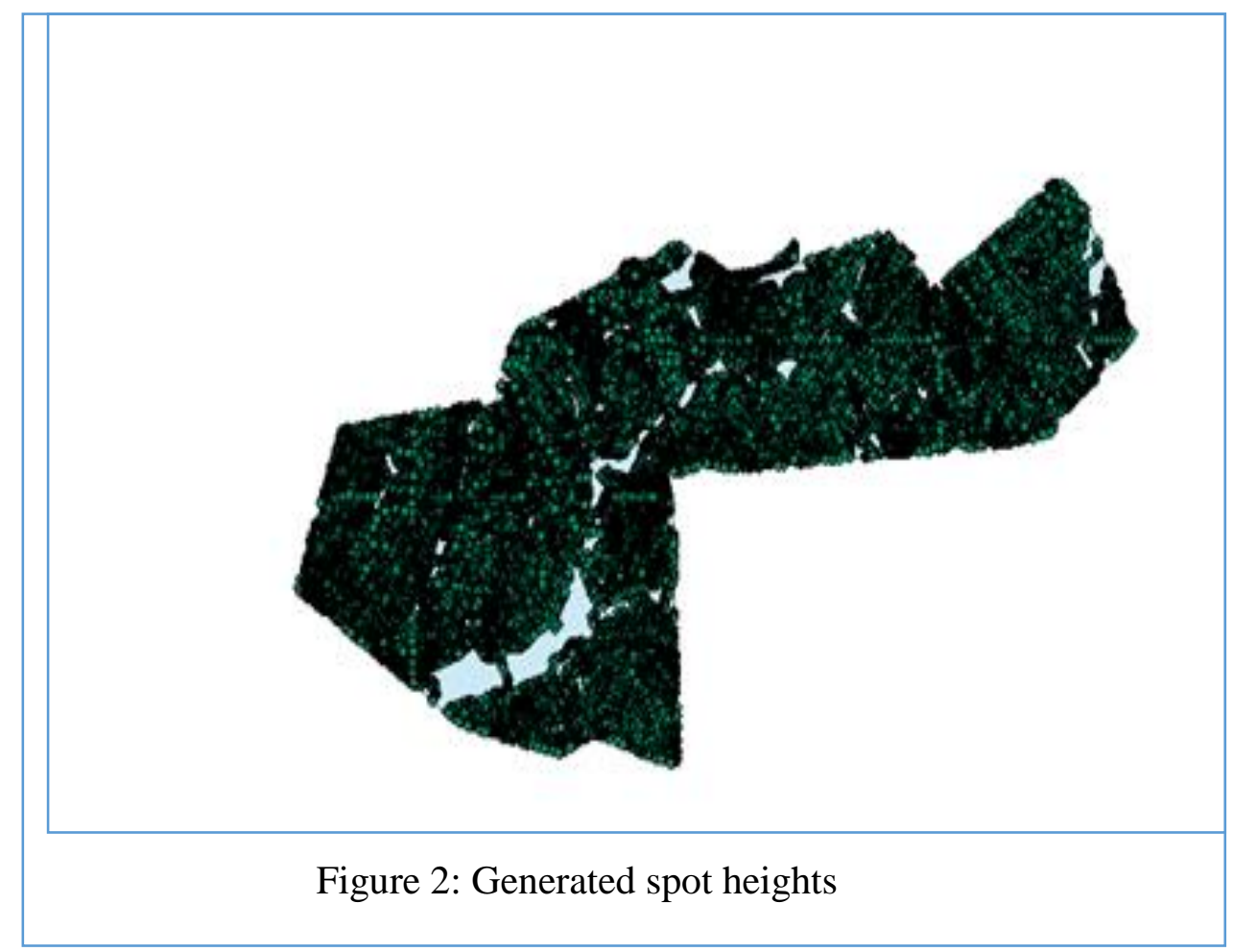

The Natural neighbor interpolation tool in spatial analyst toolbox was used to interpolate and convert the random points obtained from the contour map into a gridded surface raster. The leveled spot heights coverage of the area was overlaid on the raster surface map to enable corresponding values from the surface map to be extracted to points. As such, values from each surface matching point were extracted with its matching values from the leveled dataset.

For the Mathematical Modeling, the extracted crude heights were used as the predictors in Minitab with the real heights used as response variable. Both linear and nonlinear regressions were evaluated to select the best model. Again a multiple regression model that uses more than one response was also investigated. In this instance, the Residuals between real values and crude values were used as the response and the northing $(\mathrm{X})$ and easting $(\mathrm{Y})$ coordinates of the points used as the predictors.

Thompson's model is given by, $h r-h c=a_{0}+a_{1} x+a_{2} y+a_{3} x y+a_{4} x^{2} \ldots \ldots \ldots$ [3] (Thompson, 1968), where $h_{r}$ is the refined height and $h_{c}$ is crude height, $a_{0}, a_{1}, a_{2} \ldots$ are parameters to be determined. 
This model was fitted to the data using the least squares algorithm. Since this gave better accuracy, its results were used to develop a correction model program for converting extracted crude heights to corrected leveled heights in visual basics.

\section{Results and Discussions}

Though several mathematical models were experimented with, two models stood out as best for simulating the relationship between the leveled and extracted heights. These were the polynomial regression model and a multiple regression model of the form proposed by Thompson, (1968).

The best polynomial regression model obtained was a third degree one of form: $Z=10385-118.7 Z_{1}+0.4597 Z_{1}{ }^{2}-0.000588 Z_{1}{ }^{3}$

where $\mathrm{Z}$ is the value of response (leveled spot height) and $Z_{1}$, the value of the predictors' variables (extracted height)

The results for the fitted line of real heights $(Z)$ against extracted heights $\left(Z_{1}\right)$ are depicted in Figure 3. This shows most values clustered around the estimated trend line with a coefficient of determination of $95.9 \%$ and the sum of square errors (SSE) of $1.29 \mathrm{~m}$. 


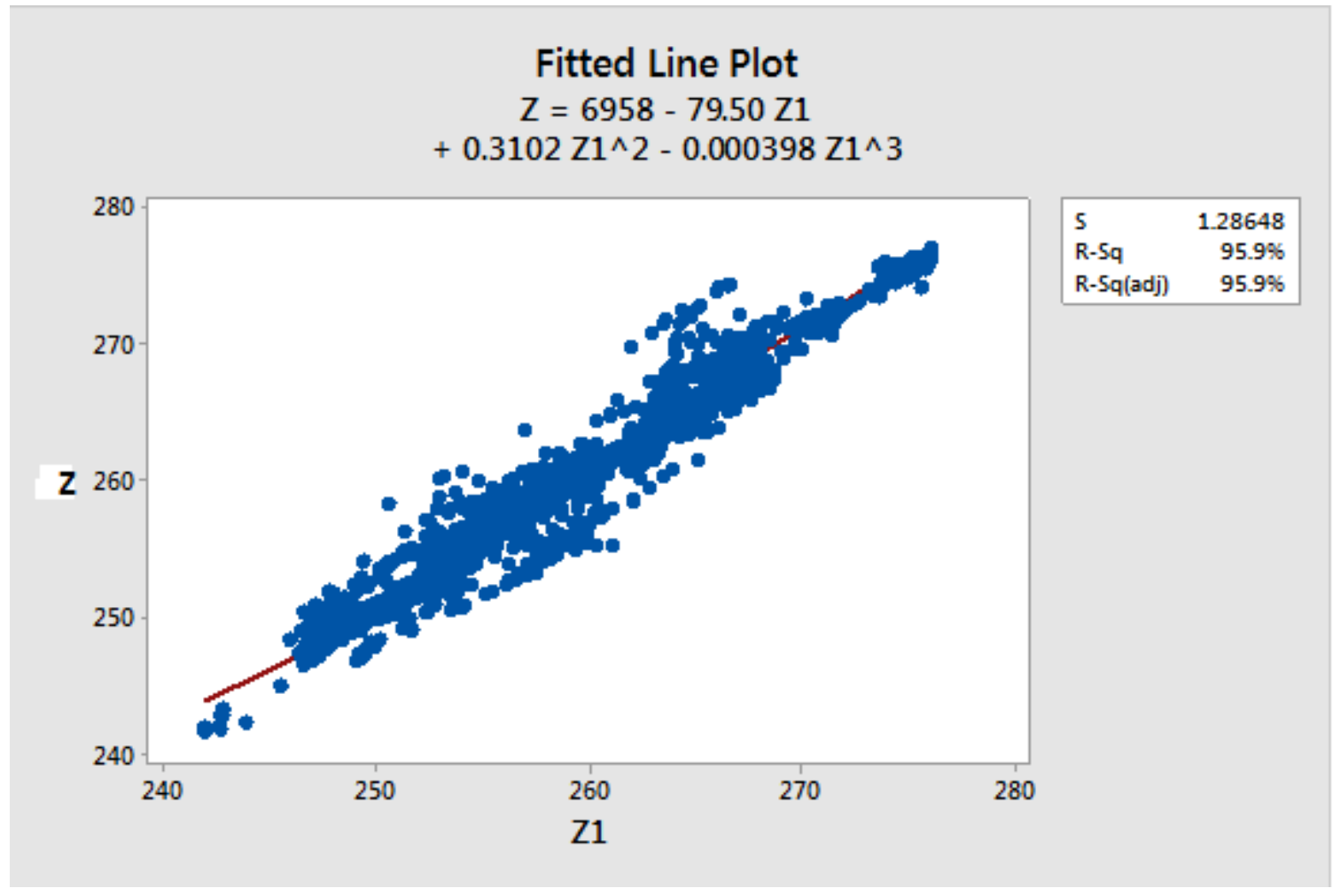

Figure 3: Graph of Real Heights against Extracted Height

Figure 4 shows a plot of residuals against the trend values. As seen, most of the residuals are clustered around the trend line even though some few appear unusually far from it. This result shows that there may be the need to investigate further the particular data values that gave these large deviations. 


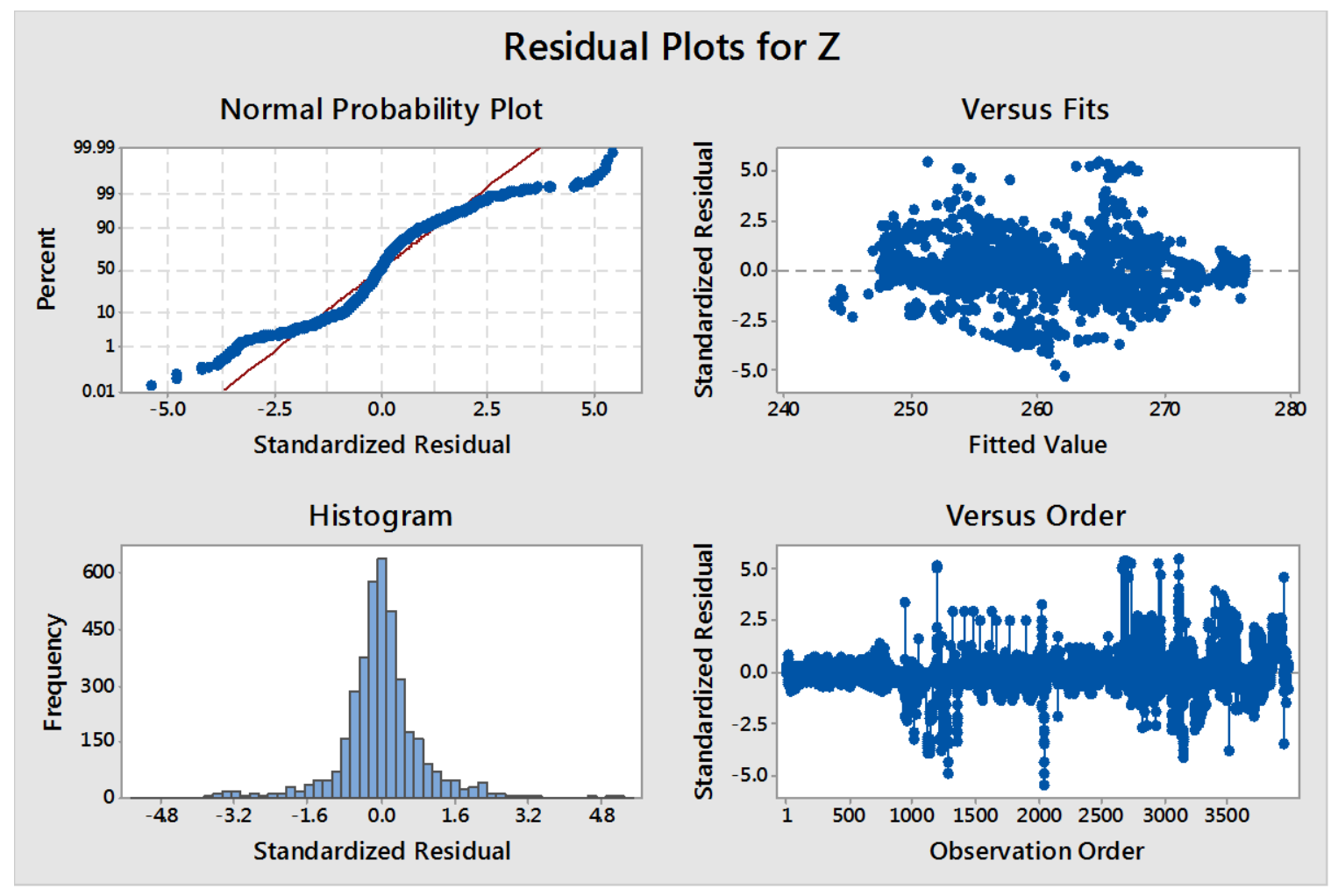

Figure 4: Residual plots for Z

The Thompson's model, which is a combination of parabolic and hyperbolic factors, was evaluated as: $h_{r}-h_{c}=29439.11392-0.156711531 x-0.11225851 y+4.99002 \times 10^{-07} x y+$ $1.14995 \times 10^{-07} x^{2}$ [5]

with a standard error of $\pm 1.3 \mathrm{~m}$.

The crude height refiner based on the Thompsons model above may receive any crude height extracted from the topographic map together with its positional coordinates to return a refined height.

\section{Model Validation.}

Validation results from a hundred crude (extracted) heights converted with the crude height convertor based on Thompson's model equation [5] and compared with test data not used in the model definition gave results that show a standard deviation of $\pm 0.79 \mathrm{~m}$. The significant 
observation of the validation results was that even those crude values that show extremely large variations from reduced levels of up to $1.47 \mathrm{~m}$ were refined to within $1.10 \mathrm{~m}$.

\section{Conclusions and Recommendations}

The research did establish that extracted spot heights from contours may not exactly match actual leveled heights on the ground but it is possible to model correction to convert such extracted heights for them to satisfactorily match actual ground reduced levels. The research achieved a method of interpolating spot heights at grid points from topographic maps using natural neighbor interpolation techniques and found that Thompson's polynomial model appears better suited for modeling differences between these and ground reduced levels. It is acknowledged that different terrain types other than what was used in this study may require a different interpolation than the nearest neighbour used and this could well be subject for research. A conversion program based on this model developed receives extracted heights and return true heights. Improvements to the Thompson's polynomial or the solutions of the polynomial itself is recommended using new techniques for solving systems of polynomial equations such as Grobner or Buchberger's algorithm (Martin et al., 2009) or Rational Function Models (RFM) (Xianyong and Xiuxiao , 2008), or else through other numerical methods.

\section{References.}

Bater, C. W., Coops , N. C. (2009). Evaluating error associated with lidar-derived DEM interpolation. Computers \& Geosciences, Vol.35, 289-300.

Childs, C. (2004). Interpolation surfaces in ArcGIS Spatial analyst. ESRI Education Services

Collins , F. C. (1995). A Comparison of Spatial Interpolation Techniques in Temperature Estimation. Blacksburg, VA.: Virginia Polytechnic Institute and State University.

Englund, E. , Sparks, A. (1988). Geo-EAS (Geostatistical Environment Assessment Software) Las Vegas , NY, U. S : Environmental Protection Agency EPA/600/4.88/033a .

Erdogan, S. (2009). A comparison of interpolation methods for producing digital elevation models at the field scale. In Earth Surface Processes and Landforms (Vol. 34, pp. 366-376).

Fisher, P.F.,Tate N.J. (2006). Causes and consequences of error in digital elevation models. Progress in Physical Geography, 30(4), 467-489.

Godone D., Garnero G. (2013). The role of morphometric parameters in Digital Terrain Models interpolation accuracy. European Journal of Remote Sensing, 46, 198-214. 
Gold , C. M. (1989). Surface Interpolation, spatial adjacency and GIS. (J. Raper, Ed.) Taylor \& Francis.

Johnston K.,Ver Hoef J.M.,Krivoruchko K., Lucas N. (2003). ArcGIS9,Using ArcGIS Geostatistical Analyst. Environmental Research Institute.

Martin Byrod,Klas Josphine,Kalle Astrom. (2009). Fast and Stable Polynomial Equation Solving and its application to computer vision, International Journal of Computer Vision. 84( 3), 237-256.

McChesney, R., McSweeney, K. (2005). Topographic Maps:Rediscovering an accessible data source for land cover change Research. Journal of Geography, 104(4), 161-178.

Methey, B. (1970). Heights from parallax bar and computer. The Photogrammetric Record. 6(35), 459465.

Sambridge , M., Braun , J., McQueen , H. (1995). Geophysical parameterization and interpolation of irregular data using natural neighbours. Geophysical Journal International, 122:837-857.

Sibson, R. (1981). A brief description of natural neighbour interpolation. In V Barnett, editor,Interpreting Multivariate Data, 21-36. Wiley, New York, U.S.A.

Thompson, E. H. (1968). Corrections to x-parallaxes. The Photogrammetric Records. 6(32) (1968), pp. 202-210

Tomlison, R. (2007). Thinking about GIS. In Geographic Information System Planning for Managers (p. 224).

Watson, D.F.,Philip G. (1987). Neighborhood-Based Interpolation. Geobyte, 2(2), 12-16.

Xianyong Lin,Xiuxiao Yuan. (2008). Improvement of the stability solving rational polynomial coefficients. International Archives of the Photogrammetry, Remote Sensing and Spatial Information Science., vol XXXVII. 